\title{
Luminosity Limit from Bound-Free Pair Production in the LHC
}

\author{
R. Bruce, S. Gilardoni, J.M. Jowett \\ CERN, Geneva, Switzerland
}

\begin{abstract}
The luminosity of the LHC as a lead-ion collider is known to be limited by the large cross-sections for electromagnetic processes in ultra-peripheral collisions. In particular, the process of bound-free $\mathrm{e}^{-} \mathrm{e}^{+}$pair production creates secondary beams of $\mathrm{Pb}^{81+}$ ions emerging from the collision points and impinging on the vacuum envelope inside superconducting magnets. New Monte-Carlo simulations, exploiting recent implementations of the physics of ion interactions with matter, are helping us to quantify the relationships among luminosity, energy deposition in the magnet coils a view to predicting and alleviating the quench limit on luminosity.
\end{abstract}




\title{
LUMINOSITY LIMIT FROM BOUND-FREE PAIR PRODUCTION IN THE LHC
}

\author{
J.M. Jowett, R. Bruce , S. Gilardoni, CERN, Geneva, Switzerland
}

\begin{abstract}
The luminosity of the LHC as a lead-ion collider is known to be limited by the large cross-sections for electromagnetic processes in ultra-peripheral collisions. In particular, the process of bound-free $\mathrm{e}^{-} \mathrm{e}^{+}$pair production creates secondary beams of $\mathrm{Pb}^{81+}$ ions emerging from the collision points and impinging on the vacuum envelope inside superconducting magnets. New Monte-Carlo simulations, exploiting recent implementations of the physics of ion interactions with matter, are helping us to quantify the relationships among luminosity and energy deposition in the magnet coils a view to predicting and alleviating the quench limit on luminosity.
\end{abstract}

\section{INTRODUCTION}

The LHC will accelerate fully stripped lead ions, $\mathrm{Pb}^{82+}$, $[1,2,3]$ with the nominal parameters and performance summarised in Table 1. For the first year or two, the number of bunches will be lower and the effect described in this paper will not limit its performance.

Table 1: LHC nominal Pb-ion parameters in collision

\begin{tabular}{|l|l|}
\hline Beam parameters & Collision \\
\hline Lead ion energy & $0.574 \mathrm{PeV}$ \\
\hline Lead ion energy/nucleon & $2.759 \mathrm{TeV}$ \\
\hline Ions/bunch & $710^{7}$ \\
\hline Number of bunches & 592 \\
\hline Stored energy per beam & $3.81 \mathrm{MJ}$ \\
\hline Peak Luminosity at IP2 & $110^{27} \mathrm{~cm}^{-2} \mathrm{~s}^{-1}$ \\
\hline
\end{tabular}

The cross section for hadronic interactions between colliding lead ions in the LHC, some 8 barn, is just a small fraction of the total cross-section for processes that modify the mass or charge of an ion sufficiently to remove it from the beam [4,5,2,3]. Most reactions occurring at the interaction point (IP) are electromagnetic processes such as copious $\mathrm{e}^{+} \mathrm{e}^{-}$pair creation and electromagnetic dissociation of the nuclei. While the majority of pair-creation events do not change the state of the ions, there is a small fraction in which a quasi-real photon converts into an electron-position pair and the electron is trapped in one of the atomic shells of one ion. This bound-free pair production (BFPP)

$$
{ }^{208} \mathrm{~Pb}^{82+}+{ }^{208} \mathrm{~Pb}^{82+} \stackrel{\gamma}{\longrightarrow}{ }^{208} \mathrm{~Pb}^{82+}+{ }^{208} \mathrm{~Pb}^{81+}+\mathrm{e}^{+}
$$

has a cross-section of $\sim 281 \mathrm{~b}$ [6] and results in a change in magnetic rigidity of the ion $\delta_{p}=1 /(Z-1)=0.012$ $[5,2]$. The $\mathrm{Pb}^{81+}$ ions form well-defined secondary beams emerging from each side of the IP.

\section{ENERGY DEPOSITION FROM BFPP}

Tracking of the $\mathrm{Pb}^{82+}$ and $\mathrm{Pb}^{81+}$ beams in the standard LHC lattice [1,3] shows that the $\mathrm{Pb}^{81+}$ beam hits the beam screen of a superconducting dipole (MB) of the dispersion suppressor, at about $380 \mathrm{~m}$ from the interaction point. The flux of $\mathrm{Pb}^{81+}$ ions is quite large: about $2.81 \times 10^{5} \mathrm{~Hz}$ at the nominal luminosity and collision energy. Therefore a detailed simulation of the energy deposition in the magnet is essential to understand the possibility of quenching the irradiated magnet.

\section{Simulation details}

The simulation of the $\mathrm{Pb}^{81+}$ ion losses has been divided into two parts: first $\mathrm{Pb}^{81+}$ ions are transported in the standard LHC optics at collision energy to determine the loss location and the ion spot size on the beam screen [3]; then a Monte-Carlo is used to evaluate the energy deposition in the superconducting coils of the dipole.

The BFPP process at the IP is not simulated, in the sense that the flux of $\mathrm{Pb}^{81+}$ is computed directly from the production cross section of 281 barn obtained by summing values from [6] over the atomic shells, and multiplied by the LHC nominal luminosity of $L=10^{27} \mathrm{~cm}^{-2} \mathrm{~s}^{-1}$. The ion production angle, at such high energies, is peaked in the forward direction and the initial angular distribution in the laboratory frame can be neglected. The spot on the beam screen is hence defined only by the optics between the IP and the dipole itself.

The tracking of ions indicates that the spot size on the beam screen typically has a transverse Gaussian distribution with a sigma of $2 \mathrm{~mm}$, a longitudinal extension of $55 \mathrm{~cm}$ (1 sigma, Gaussian) for an incident angle of 0.5 mrad.

The FLUKA Monte-Carlo program [7] is used to simulate the ion-matter interactions and to evaluate the energy losses induced in the superconducting coils by the secondary particle shower. Figure 1 shows the transverse section of the MB, which has been implemented in the FLUKA model. The simulation also includes the nominal dipole magnetic field at collision energy (8.33 T), since the hadronic and electromagnetic showers develop inside the superconducting cables and they are strongly influenced by the field distribution. 

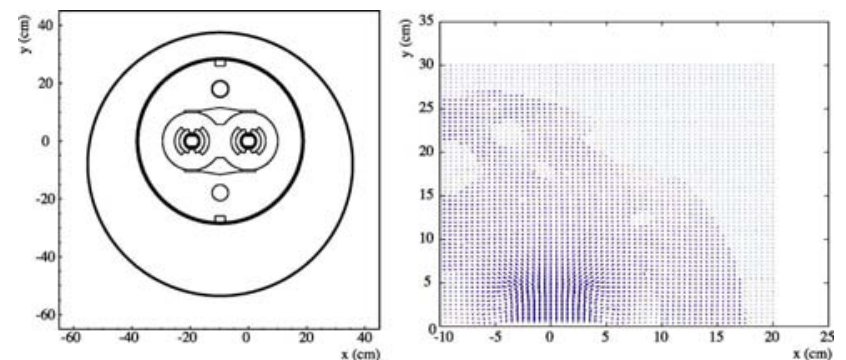

Figure 1 Transverse section of a dipole (left) and one quarter of the dipole magnetic field map (right).

\section{Simulation results}

An example of the energy deposition in the various materials of the MB is shown in Figure 2. To evaluate the energy deposition, the geometry is divided into a mesh of volumes, which are used to average and score the energy deposition itself and other relevant quantities like particle fluxes. For this study, taking account of the cylindrical symmetry of the problem, the representative volume is a sector with a radial dimension of $1.5 \mathrm{~cm}$ corresponding to the thickness of one of the superconducting coils, a length of $5 \mathrm{~cm}$ and azimuthal angle of 4 degrees.

The maximum energy deposition is then evaluated to be about $7.2 \mathrm{~mW} / \mathrm{cm}^{3}$, exceeding the quench limit of $4.5 \mathrm{~mW} / \mathrm{cm}^{3}$ given in [8] by about $60 \%$. However this comparison is biased by two assumptions: First, the heating and cooling conditions in which the $4.5 \mathrm{~mW} / \mathrm{cm}^{3}$ limit was determined are different for the case of either proton or ion losses in the operating accelerator. Secondly, the choice of the representative volume is quite delicate since it strongly influences the absolute value of the energy deposition. Quantities scored in too small a volume exhibit large statistical fluctuations due to the finite number of primary particles that can be followed by a Monte-Carlo. Quantities scored in too large a volumein the limit the complete dipole-are too diluted to be properly representative of the local interactions. For these reasons, the volume has been chosen according to the following criteria:

- It should reproduce precisely enough the particlematter interaction. Being limited by the finite number of primary particles in the simulation, the typical dimension should be of the order of the hadronic interaction length, about $15 \mathrm{~cm}$ for the coil material.

- To represent the superconducting properties of the coils, the typical volume should enclose a region that can be considered as a single thermal body.

The possibility of quenching the MB with protons has already been considered in [8] for short losses, basically following the same philosophy. However, for this specific case, losses induced by ions in a well-defined location, a different volume binning may be appropriate. Ions tend to lose energy more rapidly than protons because of their high charge state; hence most of the initial energy deposited in matter is produced by electromagnetic interactions and more localized. This would suggest a

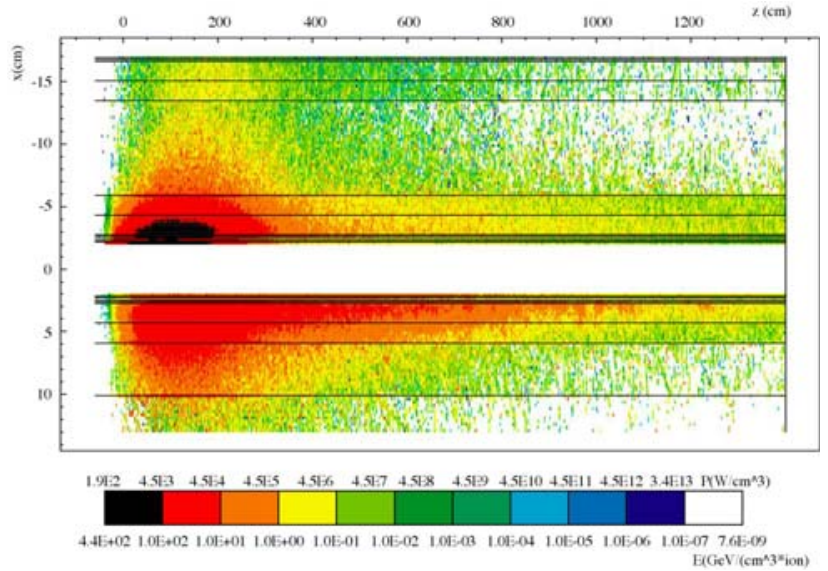

Figure 2 Longitudinal projection of the energy deposition in the dipole from ion losses. The white part is one of the two magnet apertures (the other is above). The mesh is smaller than the one described in the text.

reduction of the volume used for the proton case, in particular for the direction parallel to the shower development, reducing the $10 \mathrm{~cm}$ proposed in [8] to $5 \mathrm{~cm}$.

\section{ENERGY MARGIN ANALYSIS}

A first attempt has been made to better understand the reaction of the superconducting magnet to the ion losses. In particular, as primary goal, losses are considered on a very short timescale, before the flow of the helium in the cooling system starts to play a significant role; otherwise the thermal behaviour of the magnet would require a complete modelling of the heat flow. The following analysis applies to the first few ms after the beams start to collide and create $\mathrm{Pb}^{81+}$ ions.

The temperature margin of the NbTi conductors has been determined by [9] with the ROXIE program [10] and represents the maximum temperature increase from the $1.9 \mathrm{~K}$ of the helium bath that the conductor can experience before losing its superconducting state. The temperature margin can be converted into an energy margin which, in turn, can be compared with the energy deposition profile obtained through the Monte Carlo simulations described above. Assuming that no cooling is present, one can thus use the temperature margin to decide the time scale on which the beam losses cause a magnet to quench.

The coils in a dipole consist of superconducting $\mathrm{NbTi}$ inserted in a Cu matrix. The copper takes up 56.6\% of the volume and the NbTi $35.4 \%$. The remaining $8 \%$ of the volume is taken up by helium and insulation. Following [8], the insulation has a specific heat close to that of NbTi and a very small relative volume. Therefore it contributes very little to the total heat reserve and can be neglected in this first estimate. The helium on the other hand contributes substantially in absorbing the added heat since in this temperature range the specific heat of helium is at least one order of magnitude larger than that of NbTi. So, although it only occupies $5 \%$ of the volume, the helium has to be taken into account. However, on very short 
timescales, $t \ll 8 \mathrm{~ms}$, the flux of heat from the wire to the helium is limited and the heat does not have enough time to spread to the helium. Thus, when looking at for instance the very first $100 \mu \mathrm{s}$, as proposed by [11], the influence of the helium can be neglected.

The temperature- and magnetic-field dependent specific heats for $\mathrm{Cu}$ and for $\mathrm{NbTi}$ are given by [8]:

$$
\begin{aligned}
& C_{V, \mathrm{Cu}}(B, T)=\gamma_{\mathrm{Cu}} T+\alpha_{\mathrm{Cu}} T^{3} \\
& C_{V, \mathrm{NbTi}}(B, T)=\gamma_{\mathrm{NbTi}} \frac{B}{B_{c 2(0)}} T+\alpha_{\mathrm{NbTi}} T^{3}
\end{aligned}
$$

which gives for values of the constants $\gamma, \alpha$ and $B_{c 2(0)}$.

The total specific heat for very short timescales is then obtained by weighting the $C_{V}$ for $\mathrm{Cu}$ and for $\mathrm{NbTi}$ according to their relative volume fractions:

$$
C_{\mathrm{V}, \mathrm{tot}}=\frac{C_{V, \mathrm{Cu}} V_{\mathrm{Cu}}+C_{V, \mathrm{NbTi}} V_{\mathrm{NbTi}}}{V_{\mathrm{Cu}}+V_{\mathrm{NbTi}}}
$$

The energy required to raise the temperature to the quench limit is:

$$
\Delta Q=\int_{T_{\text {bath }}}^{T_{\text {bath }}+T_{\text {marg }}} C_{V, \text { tot }}(B, T) d T
$$

Here $T_{\text {marg } g}$ is the temperature margin and $B$ the magnetic field in the superconducting coils. These values are given for each point in a fine grid in the magnet [9]. Thus, considering the helium bath at a working temperature of $T_{\text {bath }}=1.9 \mathrm{~K}$, an energy margin was calculated for every point in the map; this is the maximum energy that can be added per unit volume before the magnet quenches. The minimum quench margin in the whole magnet was found to be $2.276 \mathrm{~mJ} / \mathrm{cm}^{3}$. FLUKA simulations show that the highest power deposition is expected around the spot where the beam hits the magnet, i.e., in the $2 \mathrm{D}$ field map, the sector covering an angle of $2 \pi / 88$ around $-\pi$. The minimum value of the energy margin in this sector was found to be $2.672 \mathrm{~mJ} / \mathrm{cm}^{3}$. As discussed in the previous section, the power deposition from the BFPP ions has a maximum value of $7.2 \mathrm{~mW} / \mathrm{cm}^{3}$. This value is obtained when the power is averaged over a cell of the dimensions $2 \times 15.5 \times 50 \mathrm{~mm}^{3}$, which covers the whole radius of the inner coil. However, on a very short timescale, the heat does not have sufficient time to migrate radially through the wire and thus the maximum energy deposition and not the radial average should be used when determining quench limits. New FLUKA simulations show that if the radial binning is made finer and finer, the maximum will converge to $13.5 \mathrm{~mW} / \mathrm{cm}^{3}$. If this power is deposited in the coil during $100 \mu \mathrm{s}, 1.35 \cdot 10^{-6} \mathrm{~J} / \mathrm{cm}^{3}$ are deposited in the hottest part of the magnet. This value is three orders of magnitudes below the lowest energy margin. Therefore the heat deposition from BFPP does not risk quenching the magnets on the $100 \mu$ s timescale.

Indeed, even if the high heat capacity of the helium is not taken into account, the wire itself could stand this power for a time

$$
t_{\max }=\frac{2.672 \mathrm{~mJ} / \mathrm{cm}^{3}}{13.5 \mathrm{~mW} / \mathrm{cm}^{3}}=0.2 \mathrm{~s}
$$

which leaves plenty of time to dump the beam. If the helium were also taken into account, this value would certainly be much higher.

\section{CONCLUSIONS}

Bound-free pair production by colliding $\mathrm{Pb}$ beams in the LHC leads to localised losses in dipoles of the dispersion suppressors. A preliminary evaluation of the energy deposited seems to suggest that the magnet will quench. However, this result is biased by the fact that the mesh used to compute the energy deposition could be too small and hence the maximum energy deposition is averaged on a volume that may not be representative of the thermal behaviour of the magnet. The determination of the dimension of this volume has already been discussed in [8] in the case of generic losses. However, in the case of the ion beam losses due to BFPP, a detailed study of the thermal behaviour of a specific magnet with a specific distribution of energy deposited by particle losses is mandatory to understand the quench limit.

Various solutions are under study to reduce the ion losses or their effects. The most obvious would be the reduction of the peak luminosity. Normally this would be considered as a very last resort but, in fact, with three experiments taking ion collisions, it may be desirable from the point of view of maximising integrated luminosity [3]. Current studies are focused on modifying the incident ion distribution on the beam screen in order to reduce the energy density deposited in the magnet.

\section{ACKNOWLEDGMENTS}

We thank A. Ferrari, G. Smirnov and M. Magistris for FLUKA support, B. Jeanneret, S. Klein, A. Siemko, M. Giovannozzi, S. Russenschuk for fruitful discussions and the temperature margin calculations.

\section{REFERENCES}

[1] The LHC Design Report, Vol. I The LHC Main Ring, CERN-2004-003 Chapter 21 and references therein.

[2] J.M. Jowett, et al, PAC2003 TPPB029.

[3] J.M. Jowett, et al, EPAC2004 MOPLT020 and references therein.

[4] A.J. Baltz et al, Phys. Rev. E 544233 (1996).

[5] S.R. Klein, Nucl. Inst. \& Methods A 459 (2001) 51.

[6] H. Meier et al, Phys. Rev. A, 63, 032713 (2001)

[7] A. Fassò et al Proc. MonteCarlo 2000 Conference, Lisbon, October 23--26 2000, A. Kling, et al (Eds.), Springer-Verlag Berlin, p. 955-960 (2001).

[8] J.B. Jeanneret et al, LHC Project Note 44 (1996).

[9] S. Russenschuck, private communication

[10] S. Russenschuck, CERN-99-01

[11]A. Siemko，Chamonix XIV， CERN-AB-2005-01 